\title{
Open Set Face Recognition Using Transduction
}

\author{
Fayin Li and Harry Wechsler \\ Department of Computer Science \\ George Mason University \\ Fairfax, VA 22030 \\ \{fli, wechsler\}@cs.gmu.edu
}

Abstract: This paper motivates and describes a novel realization of transductive inference that can address the Open Set face recognition task. Open Set operates under the assumption that not all the test probes have mates in the gallery. It either detects the presence of some biometric signature within the gallery and finds its identity or rejects it, i.e., it provides for the "none of the above" answer. The main contribution of the paper is Open Set TCM - $\boldsymbol{k N N}$ (Transduction Confidence Machine - k Nearest Neighbors), which is suitable for multi-class authentication operational scenarios that have to include a rejection option for classes never enrolled in the gallery. Open Set TCM - kNN, driven by the relation between transduction and Kolmogorov complexity, provides a local estimation of the likelihood ratio needed for detection tasks. We provide extensive experimental data to show the feasibility, robustness, and comparative advantages of Open Set TCM - kNN on Open Set identification and watch list (surveillance) tasks using challenging FERET data. Last, we analyze the error structure driven by the fact that most of the errors in identification are due to a relatively small number of face patterns. Open Set TCM - kNN is shown to be suitable for PSEI (pattern specific error inhomogeneities) error analysis in order to identify difficult to recognize faces. PSEI analysis improves biometric performance by removing a small number of those difficult to recognize faces responsible for much of the original error in performance and/or by using data fusion.

Key Words: biometrics, confidence, credibility, data fusion, information quality, Kolmogorov complexity, face recognition, open set recognition, performance evaluation, PSEI (pattern specific error inhomogeneities), randomness deficiency, strangeness, face surveillance, (multi-class) transduction, watch list, clustering, outlier detection 


\section{Introduction}

Face recognition (Chellappa et al., 1995; Daugman, 1997; Jain et al., 1999; Zhao et al., 2003; Bolle et al., 2004; Liu and Wechsler, 2004) has become one of the major biometric technologies in use. The generic open set recognition problem, a major challenge for face recognition, operates under the assumption that not all the probes (unknown test face images) have mates (counterparts) in the gallery (of known subjects). It requires the a priori availability of a reject option to answer "none of the above". If the probe is detected rather than rejected, the face recognition engine must then identify / recognize the subject. The operational analogue for open set face recognition is the (usually small) Watch List or Surveillance task, which involves (i) negative identification ("rejection") due to the obvious fact that the large majority [almost all] of the people screened at security entry points are law abiding people, and (ii) correct identification for those that make up the watch list.

Transduction, the (non-inductive) inference methodology proposed here to address the challenges characteristic of the open set face recognition problem, is a type of local inference that moves from particular to particular (Vapnik, 1998). It addresses the small sample size problem that affects face recognition due to the lack of enough data for training and/or testing. Transductive inference, in analogy to learning from unlabeled exemplars (Mitchell, 1999), is directly related to the case when one has to classify some (unknown) test (probe) face images and the choice is among several (tentative) classifications, each of them leading to different (re)partitionings of the original ID(entity) face space. The paper introduces the Open Set TCM $\boldsymbol{k N N}$ (Transduction Confidence Machine - k Nearest Neighbors), which is a novel realization of transductive inference that is suitable for open set multi-class classification. Open Set TCM $\mathrm{kNN}$, driven by the relation between transduction and Kolmogorov complexity, provides a local estimation of the likelihood ratio required for detection tasks and provides for the needed 
rejection option. The paper provides extensive experimental data to show the feasibility, robustness, and comparative advantages of Open Set TCM - kNN against existing methods using challenging FERET data.

The outline for the paper is as follows. Sect. 2 familiarizes the reader with face recognition and the protocols used. Sects. 3 and 4 discuss the learning theory behind transduction. In particular, Sect. 4 describes the TCM (Transduction Confidence Machine) (Proedrou, 2001). Sect. 5 includes one of the main contributions of this paper, Open Set TCM-kNN. It expands on TCM and provides for a novel transductive algorithm that is suitable for the open set multi-class recognition problem that includes a rejection option. Sect. 6 describes the experimental design set up, while Sects. 7 and 8 describe the use of Open Set TCM-kNN for open set and watch list / surveillance biometric recognition tasks. Sects. 9 and 10 deal with error analysis and how to use the uneven contributions to errors made by face patterns for effective data fusion. The conclusions are presented in Sect. 11.

\section{Face Recognition Tasks and Performance Evaluation Protocols}

The generic (on-line) biometric system used herein is shown in Fig. 1. The match component compares the biometric information extracted from the sample face exemplar and the signature stored in the reference (signature) template(s). One has to compare then an output score with a predefined threshold value. The comparison may be against a single template (for verification), or against a list of candidate templates (for identification). The face space, i.e., the basis needed to generate the templates, is derived using face images acquired ahead of time and independent of those that would be later on enrolled or tested (see top of Fig. 1).

FERET (Phillips et al., 1998) and BANCA (Bailly-Bailliere et al., 2003), the standard evaluation protocols in use today, are briefly described next. FERET undertakes algorithmic (technology) and scenario evaluations. It works by considering target (gallery) $T$ and query 
(probe) $Q$ sets. The output for FERET is a full (distance) matrix $S(q, t)$, which measures the similarity between each query, $q \in Q$, and each target, $t \in T$, pair. The nearest neighbor (NN) classifier authenticates then face images using the similarity scores recorded by $S$. The availability of the matrix $S$ allows for different "virtual" experiments to be conducted when one selects the specific query $P$ and gallery $G$ as subsets of $Q$ and $T$.

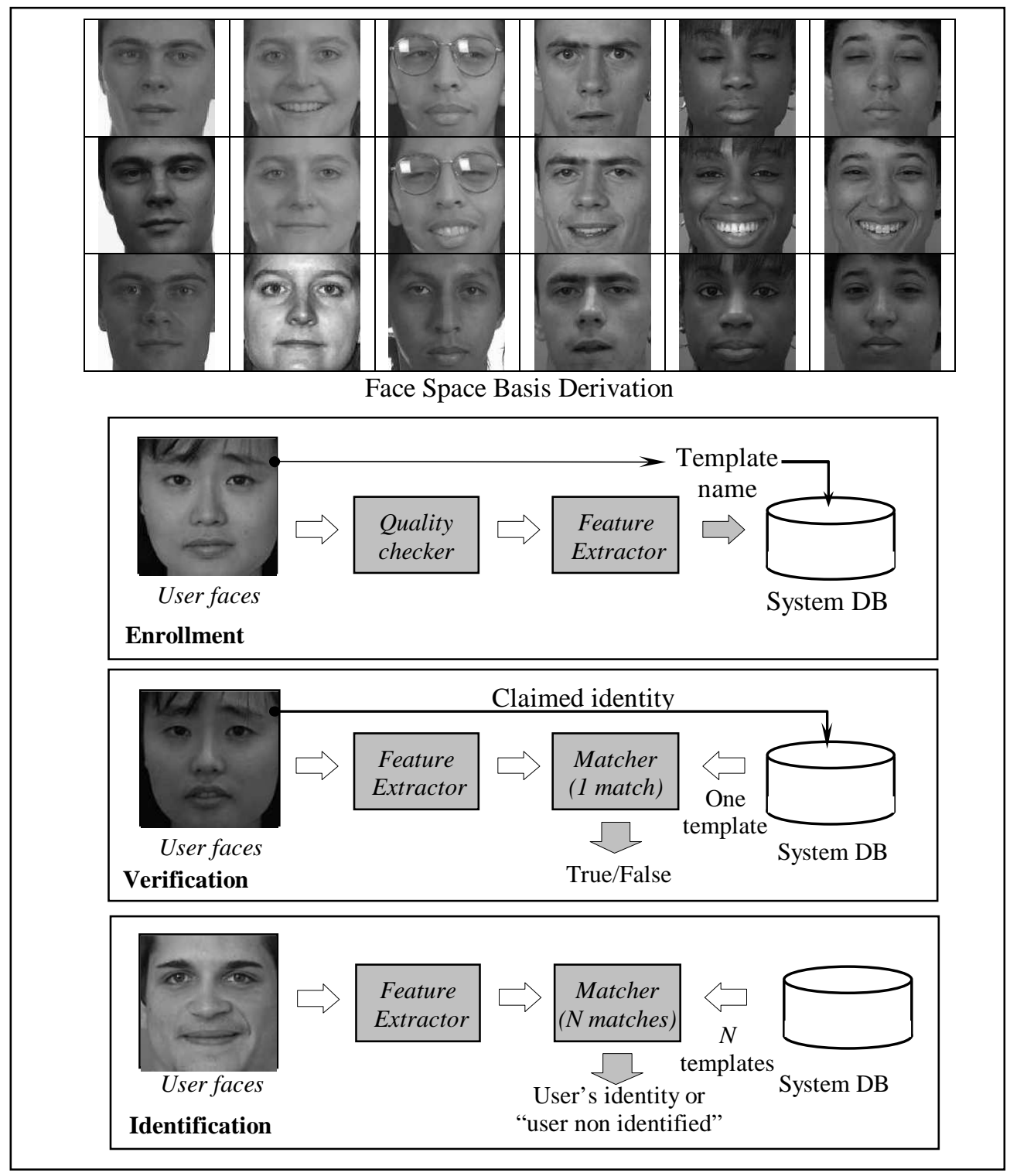

Figure 1. Face Recognition Methodology 
The 1 : N open set problem referred to by FRVT2002 (Phillips et al., 2003) as the watch list task, is briefly addressed by FRVT2002 after the two (degenerate) special cases of verification and closed set identification. Verification corresponds to an open set identification for a gallery size of $N=1$, while closed set identification seeks the match for an image whose mate is known to be in the gallery, i.e., for each image probe $p \in P$ there exists (exactly one) gallery mate $g * \in G$. Cumulative Matching Curves (CMC) and Receiver Operating Characteristics (ROC), used to display both closed and open set, are derived for different thresholds but using ground truth, which would not be available during field operation.

The closed universe model for $1: \mathrm{N}$ identification is quite restrictive as it does not reflect the intricacies for the actual real positive and negative identification scenarios. Under positive claim of identity, the user claims to be enrolled in or to be on the watch list, while under negative claim of identity the user claims not to be enrolled or known to the system. "Performance for the open set problem is quantified over two populations. First the impostors, those persons who are not present in the gallery, i.e., not on the watch list, are used to compute the false match [acceptance] rate, which is needed to quantify rejection capability. Second, for those persons who are "known" (i.e., previously enrolled) to a system, the open set identification rate, is used to quantify user [hit] performance" (Grother, 2004).

The use of the open set concept by the BANCA protocol is quite restricted. It only refers to the derivation of the feature (face) space and the parameters needed for verification. We referred to this earlier as face space basis derivation (see top of Fig. 1). BANCA protocol, however, does not address the full scope of open set identification, where some probes are not mated in the gallery.

\section{Transduction}


Transductive inference is a type of local inference that moves from particular to particular (Vapnik, 1998; 2000) (see Fig. 2). "In contrast to inductive inference where one uses given empirical data to find the approximation of a functional dependency (the inductive step [that moves from particular to general]) and then uses the obtained approximation to evaluate the values of a function at the points of interest (the deductive step [that moves from general to particular]), one estimates [using transduction] the values of a function [only] at the points of interest in one step" (Vapnik, 1998). The simplest mathematical method for transductive inference is the method of $k$ nearest neighbors. The Cover - Hart (1967) theorem proves that asymptotically the one nearest neighbor algorithm is bounded above by twice the Bayes minimum probability of error. Cover and Hart also showed that the $k$-NN error approaches the Bayes error (with factor 1) if $\mathrm{k}=\Theta(\log \mathrm{n})$.

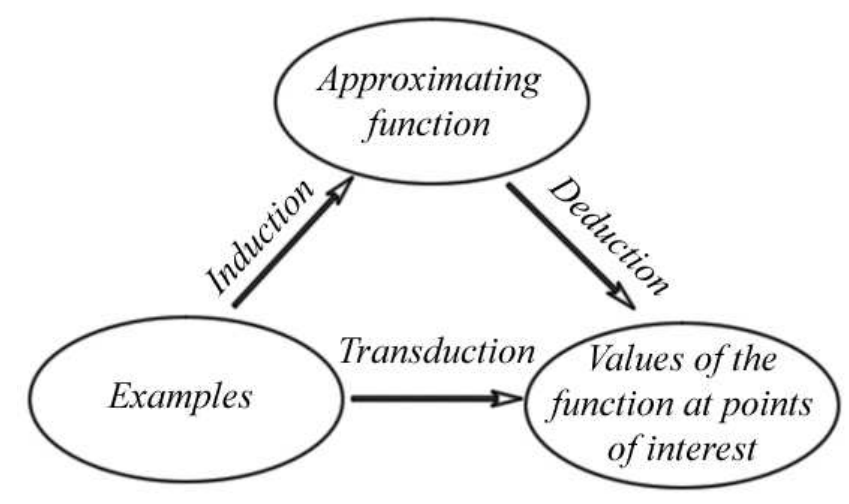

Figure 2. Transduction [Vapnik, 2000]

Vapnik (1998) approaches transductive inference as follows. Given training (labeled) exemplars one seeks among feasible labelings of the (unlabeled probe) test exemplars the one that makes the error observed during testing (labeling) consistent with the error recorded during training. It is also assumed that the training and test exemplars are i.i.d according to some [common] distribution function. Vapnik (1998) defines then the overall risk functional using two equivalent settings with the explicit goal of minimizing them. 
Setting \#1 says "Given a small sample size set of training sample exemplars, $\boldsymbol{T}$, which consists of $l$ pairs $\left(x_{\mathrm{i}}, y_{\mathrm{i}}\right)$, estimate the value of the function $y=\Phi(x)$ at the given [working test probe sample exemplars] set $\boldsymbol{W}$ of points $x_{l+1}, \ldots, x_{l+\mathrm{m}}$. (Size $l$ is considered to be small if the ratio $l / h$ is small, say $l / h<20$, where $h$ is the VC-dimension of the set). Based on the training set $T$, the working set $W$, and on a given set of functions $f(x, \alpha), \alpha \in \Lambda[\Phi(x)$ does not necessarily belong to this set], find $f\left(x, \alpha^{*}\right)$ that minimizes with a preassigned probability $1-\eta$ the overall risk of forecasting the values of the function $y_{\mathrm{i}}=\Phi\left(x_{\mathrm{i}}\right)$ on the elements of the working set $W$-- that is, which yields with probability $1-\eta$ a value of the functional

$$
R(\alpha)=\frac{1}{m} \sum_{i=1}^{m} \rho\left(y_{l+i}, f\left(x_{l+i}, \alpha\right)\right)
$$

close to the minimal one". $\rho(y, f(x, \alpha))$ is some measure of discrepancy between $y$ and $f(x, \alpha)$, say

$$
\rho\left(y_{l+\mathrm{i}}, f\left(x_{l+\mathrm{i}}, \alpha\right)\right)=\left(y_{l+\mathrm{i}}-f\left(x_{l+\mathrm{i}}, \alpha\right)\right)^{2}
$$

Setting \#1 seeks to minimize the deviation between the risk on the training and working samples.

Setting \#2 considers training and working samples [chosen according to a probability distribution function $\boldsymbol{P}(x, y)$ ], and seeks an algorithm $\boldsymbol{A}$ that will choose a function $f\left(x, \alpha_{\mathbf{A}}\right)$,

$$
f\left(x, \alpha_{\mathrm{A}}\right)=f\left(x, \alpha_{\mathrm{A}}\left(x_{1}, y_{1} ; \ldots ; x_{l}, y_{l} ; x_{l+1}, \ldots, x_{l+\mathrm{m}}\right)\right)
$$

that yields the value of the functional

$$
R(A)=\int\left(\frac{1}{m} \sum_{i=l+1}^{l+m} \rho\left(y_{i}, f\left(x_{i}, \alpha_{A}\right)\right)\right) d P\left(x_{1}, y_{1}\right) \ldots d P\left(x_{l}, y_{l}\right) d P\left(x_{l+1}, \ldots, x_{l+m}\right)
$$

close to the minimal one, when the sum $\Sigma$ is indexed for (unlabeled) exemplars $i$ between $l+1$ and $l+m$. Setting \#2 labels $W$, the working exemplars set, in a fashion consistent with $T$, the training set, e.g., using minimization for some error functional over $\mathrm{T} \cup \mathrm{W}$. One possible realization using SVM (support vector machines) is to classify the joint set of training and working exemplars with maximal margin over all possible classifications $\mathbf{L}(\mathbf{W})$ for the working set W, e.g., $\arg \min _{\mathbf{L}(\mathrm{w})} \min _{\mathrm{w}} 1 / 2\|\mathrm{w}\|^{2}$ (Saunders et al., 1999). 
The solutions for Settings \#1 and \#2 are connected (Theorem 8.1) (Vapnik, 1998). An example is TSVM (Transductive inference using SVM), which has been shown to yield substantial improvements for text classification, a two-class classification task, over inductive methods, especially for small training sets and large test (working) sets. The observed success was explained due to the fact that "the margin of separating hyperplanes is a natural way to encode prior knowledge for (text) classifiers and to study the location of text exemplars (with respect to the hyperplane), which is not possible for an inductive learner" (Joachims, 1999).

The goal for inductive learning is to generalize for any future test set, while the goal for transductive inference is to make predictions for a specific working set. The working exemplars provide additional information about the distribution of data and their explicit inclusion in the problem formulation yields better generalization on problems with insufficient labeled points (Gammerman et al., 1998). Transductive inference becomes suitable for face recognition when some of the faces available for training lack proper ID(entification) or when one has to classify some (unknown) test face image(s). The challenge is to choose among several (tentative) classifications, each of them leading to different partitionings of the ID(entity) face space. The scope for transducive inference is augmented herein in order to cope with the open set recognition problem, which requires (a) detection in addition to mere identification; and (b) the ability to cope with multi-class rather than two-class classification. The solution to this problem is presented in the next two sections.

\section{Kolmogorov Complexity and Randomness Deficiency}

There is a strong connection between transductive inference and Kolmogorov complexity. Let $\#(z)$ be the length of the binary string $z$ and $K(z)$ its Kolmogorov complexity, which is the length of the smallest program (up to an additive constant) that a Universal Turing Machine needs as input in order to output $z$. The randomness deficiency $\boldsymbol{D}(\boldsymbol{z})$ for string $z$ (Li and Vitanyi, 1997; 
Vovk et al., 1999) is

$$
\mathrm{D}(z)=\#(z)-\mathrm{K}(z)
$$

and it measures how random is the binary string $z$ and the set it represents. The larger the randomness deficiency is, the more regular and more probable the string $z$ is (Vovk et al., 1999). [Another connection between Kolmogorov complexity and randomness is via MDL (minimum description length).] Transductive inference seeks to find from all possible labelings $L(\mathrm{~W})$ the one that yields the largest randomness deficiency, i.e., the most probable labeling. This choice models the working ("test") exemplar set $W$ in a most similar fashion to the training set $T$ and would thus minimally change the original model for $T$ or expand it for $\mathrm{T} \cup \mathrm{W}$ (see Sect. 3 for the corresponding transductive settings). "The difference between the classifications that induction and transduction yield for some working exemplar approximates its randomness deficiency. The intuitive explanation is that we disturb a classifier (driven by $T$ ) by inserting a new working examplar in a training set. A magnitude of this disturbance is an estimation of the classifier's instability (unreliability) in a given region of its problem space” (Kukar and Kononenko, 2002).

Randomness deficiency is, however, not computable ( $\mathrm{Li}$ and Vitanyi, 1997). One has to approximate it instead using a slightly modified Martin- Löf test for randomness and the values taken by such randomness tests are called $\boldsymbol{p}$-values. The $\boldsymbol{p}$-value construction used here has been proposed by Gammerman et al. (1998) and Proedrou et al. (2001). Given a sequence of distances from exemplar $i$ to other exemplars, the strangeness of $i$ with putative label $y$ is defined as:

$$
\alpha_{i}=\left(\sum_{j=1}^{k} d_{i j}^{y}\right)\left(\sum_{j=1}^{k} d_{i j}^{-y}\right)^{-1}
$$

The strangeness measure $\alpha_{i}$ is the ratio of the sum of the $\boldsymbol{k}$ nearest distances $d$ from the same class $(y)$ divided by the sum of the $k$ nearest distances from all the other classes $(-\boldsymbol{y})$. The strangeness of an exemplar increases when the distance from the exemplars of the same class 
becomes larger and when the distance from the other classes becomes smaller. The smaller the strangeness the larger its randomness deficiency is. Note that each new test exemplar $\boldsymbol{e}$ with putative label $y$ and derived strangeness $\alpha_{\text {new }}^{y}$ requires to recompute, if necessary, the strangeness for all the training exemplars when the identity of their $k$ nearest neighbors exemplars changes due to the location of (the just inserted new exemplar) $\boldsymbol{e}$.

The $\boldsymbol{p}$-value construction shown below, where $\boldsymbol{l}$ is the cardinality of the training set $T$, constitutes a valid randomness (deficiency) test approximation (Melluish et al., 2001) for this tranductive (putative label $y$ ) hypothesis.

$$
p_{y}(e)=\frac{\#\left\{i: \alpha_{i} \geq \alpha_{\text {new }}^{y}\right)}{l+1}
$$

An alternative valid randomness (deficiency) approximation (Vovk et al., 1999) and the one that we use here defines the $\boldsymbol{p}$-value for a working exemplar $\boldsymbol{e}$ (with putative label $y$ ) as:

$$
p_{y}(e)=\frac{f\left(\alpha_{1}\right)+f\left(\alpha_{2}\right)+\cdots+f\left(\alpha_{l}\right)+f\left(\alpha_{\text {new }}^{y}\right)}{(l+1) f\left(\alpha_{\text {new }}^{y}\right)}
$$

where the function $f$ used is monotonic non-decreasing with $f(0)=0$, and $l$ is the number of training exemplars. Experimental data reported later in this paper uses $f(\alpha)=\alpha$. Our empirical evidence has shown that the alternative randomness approximation (8) yields better performance than the standard one (7), which may suffer from "distortion phenomenon" (Vovk et al., 1999). If there are $c$ classes in the training data, there are c $\boldsymbol{p}$-values for each working exemplar $\boldsymbol{e}$. Using $\boldsymbol{p}$ values one chooses that particular labeling driven by the largest randomness deficiency for class membership, i.e., the putative label $y$ that yields the least strangeness or correspondingly the largest $\boldsymbol{p}$-value. This largest $\boldsymbol{p}$-value is also defined as the credibility of the label chosen, which is a measure of information quality. The associated confidence measure, which is derived as the 1 st largest $\boldsymbol{p}$-value (or one) minus the 2 nd largest $\boldsymbol{p}$-value, indicates how close the first two 
assignments are. The confidence value indicates how improbable classifications other than the predicted labeling are, while the credibility value shows how suitable the training set is for the classification of that working exemplar.

The transductive inference approach uses the whole training set $T$ to infer a rule for each new exemplar. Based on the $\boldsymbol{p}$-values defined above, Proedrou et al. (2001) have proposed the TCM$\boldsymbol{k N N}$ (Transduction Confidence Machine - k Nearest Neighbor) to serve as a formal transduction inference algorithm for classification purposes. TCM-kNN has access to a distance function $\boldsymbol{d}$ that measures the similarity between any two exemplars. Different similarity measures (see Sect. 6) are used and their relative performance varies accordingly. TCM-kNN does not address, however, the detection (decision) aspect needed for open set face recognition. Our proposed solution for the detection aspect involves using the PSR (peak-side-ratio) that characterizes the distribution of $\boldsymbol{p}$-values. It implements the equivalent of the likelihood ratio $(\boldsymbol{L R})$ used in detection theory and hypothesis testing, where $L R$ is the ratio between the hypothesis $H_{0}$ that the unknown probe belongs to the gallery and $H_{1}$ (alternative hypothesis) that it does not belong.

The distribution for the PSR, if impostor cases were made available, serves to determine how to threshold in order to accept or reject a particular working exemplar $\boldsymbol{e}$. Towards that end, one would relabel the training exemplars, one at a time, with all putative labels except the one originally assigned to it. The corresponding PSR should resolve each such relabeled exemplar suitable for rejection because its new label is mistaken. The resulting distribution for the PSR determines then when to reject working exemplars as impostors. Open Set TCM - kNN implements the above concepts and it is described in the next section.

\section{Open Set TCM-kNN}

Open Set recognition operates under the assumption that not all the probes have mates in the gallery and it thus requires the reject option. Given a new (test / probe) working exemplar $\boldsymbol{e}$, the 
$\boldsymbol{p}$-values output from Open Set TCM-kNN records the likelihoods that the new exemplar comes from each putative subject in the training data. If some $\boldsymbol{p}$-value is high enough and it significantly outscores the others, the new exemplar can be mated to the corresponding subject ID with credibility $\boldsymbol{p}$. If the top ranked (highest $\boldsymbol{p}$-values) choices are very close to each other and outscore the other choices, the top choice can still be accepted but its recognition is questionable due to ambiguity and yields low confidence. The confidence measures the difference between the 1 st and 2nd largest (or consecutive) $\boldsymbol{p}$-values. If all $\boldsymbol{p}$-values are randomly distributed and no $\boldsymbol{p}$-values outscore other $\boldsymbol{p}$-values enough, any recognition choice will be questionable and the new exemplar should be rejected. The proposed PSR (peak-to-side ratio)

$$
P S R=\left(p_{\text {max }}-p_{\text {mean }}\right) / p_{\text {stdev }}
$$

characterizes those characteristics of $\boldsymbol{p}$-value distribution, where $p_{\text {mean }}$ and $p_{\text {stdev }}$ are the mean and standard deviation of the $\boldsymbol{p}$-value distribution without $p_{\max }$.

The threshold for rejection is learned a priori from the composition and structure of the training data set at enrollment time. Each training exemplar $\boldsymbol{e}$ is iteratively reassigned to all possible classes but different from its own and the $\boldsymbol{p}$-values are recomputed accordingly. The PSR is derived using the recomputed $p$-values with $\boldsymbol{e}$ playing the role of an impostor. The PSR values found for such impostors are low (since they do not mate) compared to those derived before for legitimate subjects and they require rejection. The PSR distribution (and its tail) provides a robust method for deriving a priori the operational threshold $\boldsymbol{\Theta}$ for detection as

$$
\boldsymbol{\Theta}=P S R_{\text {mean }}+3 \times P S R_{\text {stdev }}
$$

where $P S R_{\text {mean }}$ and $P S R_{\text {stdev }}$ (standard deviation) are characteristic for the $P S R$ distribution. The probe is then rejected if the relationship $\boldsymbol{P S R}_{\text {new }} \leq \boldsymbol{\Theta}$ holds true. Correspondingly, authentication takes place for (large) values exceeding $\boldsymbol{\Theta}$. 
There are conceptual similarities between the use of the PSR to approximate the likelihood ratio and scoring normalization methods used in speaker verification (Furui, 1997; Reynolds et al., 2000). The alternative hypothesis for speech is modeled using either the cohort or the universal background model (UBM). The cohort approximates the alternative $H_{1}$ hypothesis using speechspecific (same gender impostor) subjects, while UBM models $H_{1}$ by pooling speech from several speakers and training a single speaker background model. The PSR measure is conceptually related to the cohort model, as both implement $L R$ using local estimation for the alternative hypothesis. The ability of the cohort model to discriminate the speaker's speech from those of similar, same gender impostors is much better than that offered by UBM (Mak et al., 2001) and it leads to improved security at lower FAR (false acceptance rates). Similar arguments hold for other modalities, including human faces.

\section{Experimental Design}

The data set (see Fig. 3) from FERET (Phillips et al., 1998) consists of 750 frontal face images corresponding to 250 subjects. 200 subjects come from the difficult batch \#15 that was acquired using variable illumination and/or facial expressions, while the remaining different 50 subjects consists of are drawn from other batches. . Each subject has three normalized (zero mean and unit variance) images of size 150 x 150 with 256 gray scale levels. Each column in Fig. 3 corresponds to one subject. The normalized 300 face images from 100 subjects are used to generate PCA and FLD (Fisher Linear Discriminant) face basis (see top of Fig. 1). 50 subjects are randomly selected from batch \#15 and the remaining different 50 subjects were drawn from other batches. The remaining 450 face images for 150 subjects are used for enrollment and testing. They are projected on the PCA and FLD face bases derived ahead of time to yield 300 PCA coefficients and 100 Fisherfaces using FLD on the reduced 300 PCA space (Liu and Wechsler, 2002). For each subject, two images are randomly selected as training and the third one as testing. 


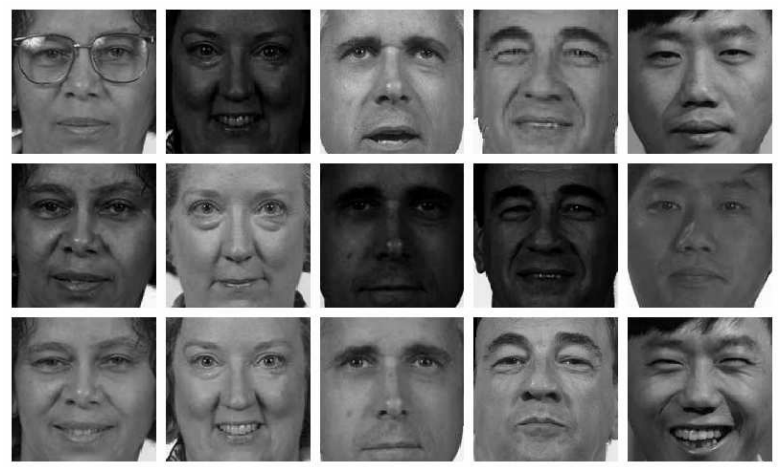

Figure 3. Face Images

We have used several well-known similarity measures (see below) to evaluate their effect on different face representation (PCA and Fisherfaces) when using TCM-kNN (k=1). The similarity distances $\boldsymbol{d}$ used are shown next. Given two $n$-dimensional vectors $X, Y \in \mathfrak{R}^{n}$, the distance measures used are defined as follows:

$$
\begin{gathered}
d_{L 1}(X, Y)=|X-Y|=\sum_{i=1}^{n}\left|X_{i}-Y_{i}\right| \quad d_{L 2}(X, Y)=\|X-Y\|^{2}=(X-Y)^{T}(X-Y) \\
d_{\text {cos }}(X, Y)=-\frac{X^{T} Y}{\|X\| Y \|} \quad d_{\text {Dice }}(X, Y)=-\frac{2 X^{T} Y}{\|X\|^{2}+\|Y\|^{2}}=-\frac{2 X^{T} Y}{X^{T} X+Y^{T} Y} \\
d_{\text {Jaccard }}(X, Y)=-\frac{X^{T} Y}{\|X\|^{2}+\|Y\|^{2}-X^{T} Y}=-\frac{X^{T} Y}{X^{T} X+Y^{T} Y-X^{T} Y} \\
d_{\text {Mah }+L 2}(X, Y)=(X-Y)^{T} \Sigma^{-1}(X-Y) \quad d_{\text {Mah }+\cos }(X, Y)=-\frac{X^{T} \Sigma^{-1} Y}{\|X \mid\| Y \|}
\end{gathered}
$$

where $\Sigma$ is the covariance matrix of the training data. For PCA, $\Sigma$ is diagonal and the diagonal elements are the (eigenvalues) variances of the corresponding components. The Mahalanobis $+L_{1}$ distance defined only for PCA is

$$
d_{M a h+L 1}(X, Y)=\sum_{i=1}^{n}\left(\frac{\left|X_{i}-Y_{i}\right|}{\sqrt{\lambda_{i}}}\right)
$$

$L_{1}$ defines the city-block distance, $L_{2}$ defines the Euclidean distance. Cosine, Dice, Overlap and Jaccard measure the relative overlay between two vectors. $L_{1}, L_{2}$ and cosine can also be weighted by the covariance matrix of training data, which leads to Mahalanobis related distances. Our empirical findings indicate that Mahalanobis related similarity distances are superior to others 
when expressive features (driven by PCA) are used; while overlay related similarity distances are superior when discriminating (Fisherfaces) features are used.

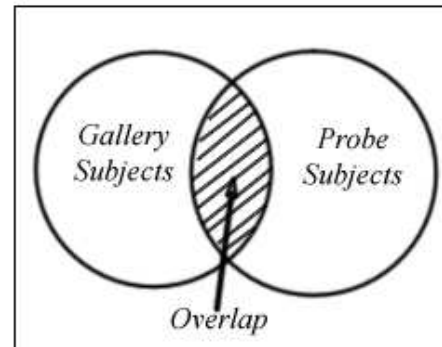

(a) Open Set

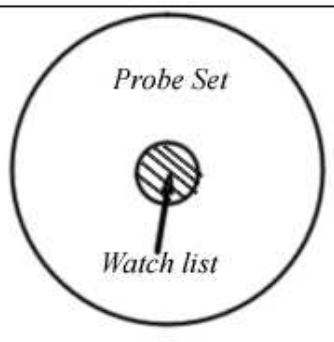

(b) Watch list

Figure 4. Generic Open Set and Watch List Tasks

The next two sections present experimental data that illustrates the usefulness and robustness of Open Set TCM - kNN for (generic) open set face recognition and watch list tasks (see Figs. $4 \mathrm{a}$ and $4 \mathrm{~b}$, respectively). Watch list, a special task for open set face recognition, corresponds to the case when the overlap between the gallery and probe sets is the gallery (watch list) itself and the probe set size is much larger than the gallery. Open set and watch list can be thought operationally relevant to the US-VISIT program where applicants are matched against large data bases to possibly avoid repeat visa applications, while watch list corresponds to the case where subjects are matched for negative identification against some WANTED list.

\section{Open Set Face Recognition}

Biometric systems in general, and face recognition engines, in particular, require significant tuning and calibration, for setting the detection thresholds among other things, before "plug and play". Setting thresholds is not easy to automate due to their strong dependency on image quality and the composition of training data. Note also that "much more is known about the population, [or genuine customers,] of an application than is known about the enemies, [i.e., the imposters that have to be rejected]. Consequently, the probability of a false alarm rate (FAR), a false match [for 
screening and positive identification], is hard to estimate. Hence, the false reject rate (FRR), that concerns open set negative identification, is easier to estimate than the false alarm rate, because the biometric samples of the enemy population are not available" (Bolle et al., 2004). The thresholds needed for field deployment have to be set up ahead of time, i.e., a priori, and without resorting to additional client, e.g., impostor data. The alternative of setting the thresholds $\boldsymbol{a}$ posteriori using the ground truth available from the aggregate similarity scores recorded for matching the probe set against the gallery set is not appropriate because the ground truth is not available.

Conventional threshold selection methods typically compute the distribution of inter- and intra ID (subject) distances, and then choose a threshold to equalize the overlapping areas of the distributions, i.e., to equalize the false acceptance rate (FAR) and false rejection rate (FRR). "The success of this approach, however, relies on whether the estimated distributions match the subject- and impostor-class distributions. Session-to-session ID variability, however, contributes much bias to the thresholds, rendering the authentication system unstable" (Bengio et al., 2001).

Comparative performance of Open Set TCM - kNN against Open Set $\{$ PCA, Fisherfaces $\}$, the corresponding versions for PCA and Fisherfaces, which are standard and well known face recognition methods, is presented next. The face space basis derivation is done before enrollment (see Fig. 1), the corresponding data collection was described in Sect. 6, and nearest neighbor identification is done using the distances described in the previous section. Detection thresholds for Open Set TCM - kNN are found as described in Sect. 5, while detection thresholds for Open Set $\{$ PCA and Fisherfaces $\}$ are found as explained next.

The Open Set standard PCA ("Eigenfaces") and Fisherfaces classifiers derive their rejection threshold from the intra- and inter-distance (similarity) distribution of training exemplars in a fashion similar to that used by FRVT2002. The statistics of intra-distance ("within") distribution 
set the lower bound of the threshold and the statistics of inter-distance ("between") distribution set the upper bound. As the minimum distance of the new (test / probe) exemplar to the prototypes for each class becomes closer to or larger than the upper bound, the more likely the new testing exemplar will be rejected. Our experiments have shown that face recognition performance varies according to the threshold chosen.

The recognition rate reported is the percentage of subjects whose probe is correctly recognized or rejected. Faces are represented using either 300 PCA or 100 Fisherfaces components. From the 150 subjects available (see Sect. 6), 80 subjects are randomly selected to form a fixed gallery, while another 80 subjects are randomly selected as probes such that 40 of them have mates in the gallery, i.e, the gallery and probe sets have an overlap of 40 subjects. The gallery consists of two (out of 3) randomly selected images; while the probes consist of the remaining one (out of 3 ) images for faces that belong to the gallery and one (out of 3) randomly selected image for subjects that do not belong to the gallery. During testing, all distance measurements from Sect. 6 are used and the threshold varies from lower to upper bound. The same experiment is run 100 times for different probe sets. The distance measurements $d$ for Open Set $\{$ PCA and Fisherfaces $\}$ that yield the best results are Mahalanobis $+L_{2}$ and cosine, respectively. Fig. 5 shows the mean recognition rate for different thresholds. When ground truth is available the thresholds $\boldsymbol{\Theta}$ are optimally set to yield maximum performance, and the reject decision is taken if $(\mathrm{min}) \mathrm{d}>\boldsymbol{\Theta}$ reject. The best average (over 100 experiments) authentication (correct rejection and identification) rates (see Fig. 5) for Open Set $\{$ PCA, Fisherfaces $\}$ classifiers that yield $\mathrm{FAR}=7 \%$ are:

- $74.3 \%$ (s.d. $=3.06 \%$ ) for PCA representation and sometime the optimal $\boldsymbol{\Theta} \sim\left(\right.$ Intra $_{\text {mean }} \times$

$$
\text { Intra } \left.a_{\text {stdev }}+\text { Inter }_{\text {mean }} \times \text { Inter }_{\text {stdev }}\right) /\left(\text { Inter }_{\text {stdev }}+\text { Intra }_{\text {stdev }}\right)
$$


- $85.4 \%($ s.d. $=2.30 \%)$ for Fisherfaces representation and sometime the optimal $\boldsymbol{\Theta} \sim$ $\left(\right.$ Intra $_{\text {mean }} \times$ Inter $_{\text {stdev }}+$ Inter $_{\text {mean }} \times$ Intra $\left._{\text {stdev }}\right) /\left(\right.$ Inter $_{\text {stdev }}+$ Intra $\left._{\text {stdev }}\right)$.
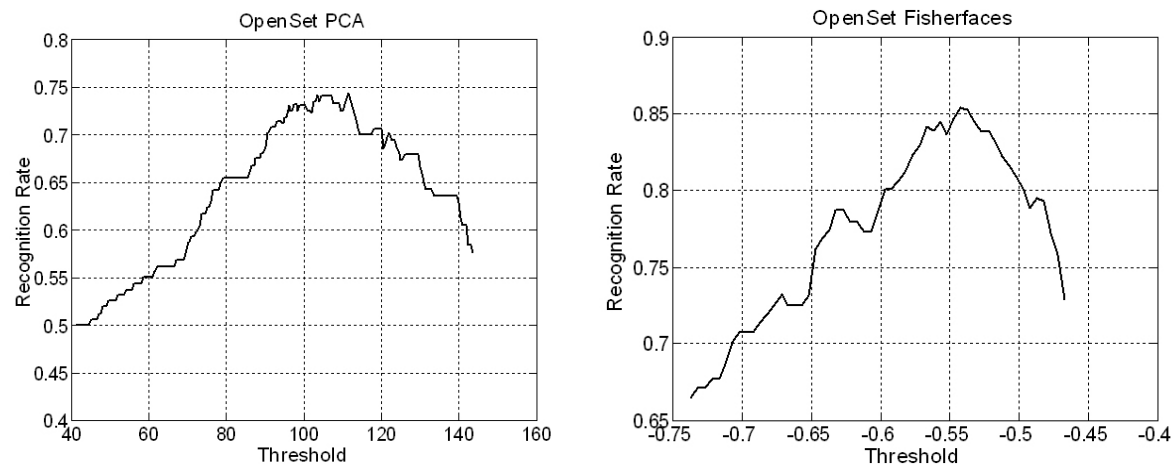

Figure 5. The Recognition Rate vs Threshold: PCA (Left) and Fisherfaces (Right)

For Open Set PCA, the results are very close if the number of components used varies from 150 to 300, while for Open Set Fisherfaces, the results are very close if the number of components used varies from 55 to 90 . More experiments have been done randomly varying the gallery set and similar results were obtained. The optimal threshold, however, varies largely with the gallery set and probe, and would be hard to be determined a priori. Attempts made to learn the threshold a priori, i.e., without ground truth knowledge were unsuccessful.
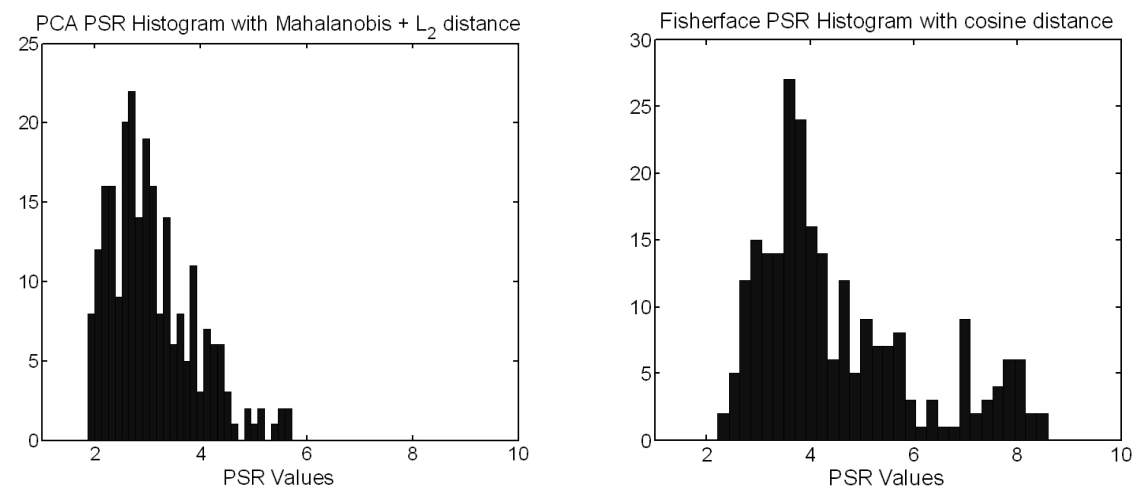

Figure 6. PSR Histogram for PCA (Left) and Fisherfaces (Right) Components

The same Open Set experiment was run then using Open Set TCM $-\mathrm{kNN}$ for $\mathrm{k}=1$. The only difference now is that the rejection threshold $\boldsymbol{\Theta}$ is computed a priori according to the $P S R$ 
procedure described in Sect. 5 (see Fig. 6) Authentication is driven by large PSR and the average authentication (correct rejection and identification) rates for (measured) FAR $=6 \%$ are:

- $81.2 \%$ (s.d. $=3.1 \%$ ) for PCA using $\boldsymbol{\Theta}=5.51$ and the Mahalanobis $+L_{2}$ distance;

- $88.5 \%$ (s.d. $=2.6 \%$ ) for Fisherfaces using $\boldsymbol{\Theta}=9.19$ and the cosine distance.

Using PCA, the results for Open Set TCM-kNN are very close if the number of components used varies from 170 to 300, while using Fisherfaces the results for Open Set TCM-kNN are very close if the number of components used varies from 55 to 80 . More experiments have been done randomly varying the gallery set and similar results are obtained. The threshold varies with the chosen gallery set and is determined a priori. This is different from Open Set $\{$ PCA, Fisherfaces where the performance shown is obtained only if the thresholds were optimally set a posteriori using ground truth. Keeping this significant difference in mind, Open Set TCM-kNN outperforms the Open Set $\{$ PCA, Fisherface $\}$ classifiers. Attempts to set the thresholds ahead of time ("a priori”) for the Open Set $\{$ PCA, Fisherfaces $\}$ classifiers were not successful, because the intraand inter- distance distributions for the gallery are not too powerful to characterize the behavior of the probe.

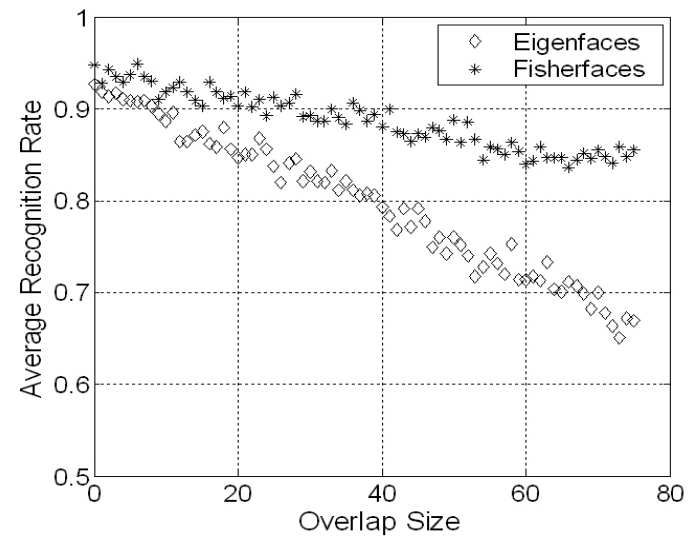

Figure 7. Mean Detection and Identification Rates vs. Overlap Size

The next experiment assesses how the overlap size between the gallery and probe sets affects open set performance and the role different representations play. Fig. 7 shows the mean detection 
and recognition rates for Open Set TCM - kNN using PCA and Fisherfaces representations, and the Mahalanobis $+L_{2}$ and cosine distances, respectively. There are 150 subjects available, the size for both the gallery and the probe sets is 75 subjects, and the overlap between the gallery list and the probe set varies from 0 to 75 subjects. We report the average results obtained over 100 randomized (over gallery and probe composition) runs. The performance goes down, almost linearly, as the overlap size increases. Fisherfaces components yield overall much better performance compared to PCA components, except for very small overlap size when the performance observed is closed but still better when using Fisherfaces than PCA components. The explanation for the observed performance is that as the size of overlap increases, it becomes more difficult to detect and identify individuals on overlap set. The performance for the Open Set $\{$ PCA, Fisherfaces $\}$ classifiers was very poor.
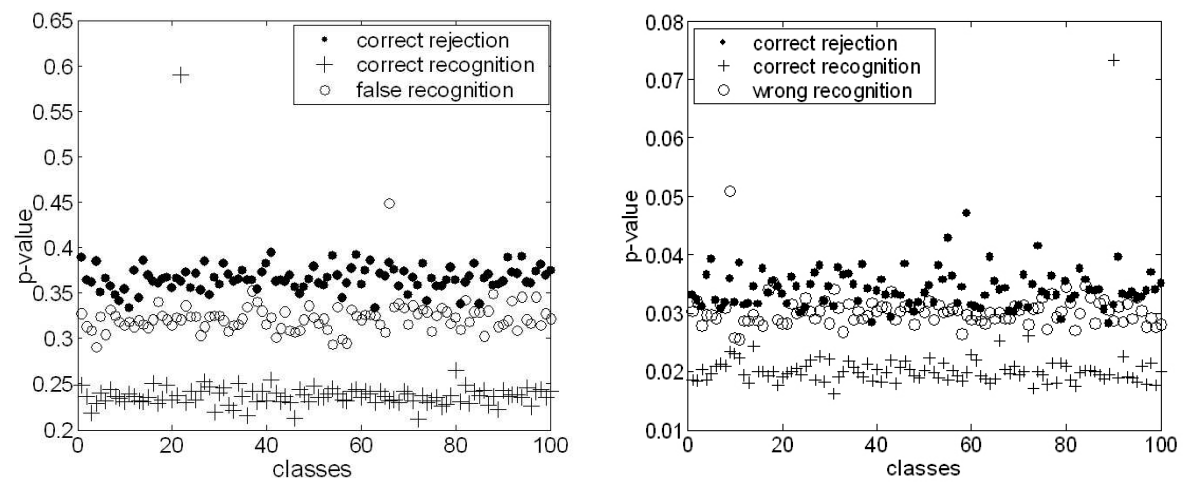

Figure 8. p-value Distribution of Rejection, Correct and False Recognition Using PCA with (Mahalanobis $\left.+L_{2}\right)$ Distance (Left) and Fisherfaces with Cosine Distance (Right)

Open Set TCM - kNN also provides measures of credibility and confidence concerning the recognition decisions it makes. The $\boldsymbol{p}$-value distribution behind Open Set TCM - kNN shows (see Fig. 8) its ability for detection and authentication [see non-overlapping correct rejection $(\bullet)$ and correct recognition (+) layers separated by the false recognition (o) layer] and supports the use of PSR measurement for rejection. 


\section{Watch List}

The gallery of wanted individuals is now very small compared to the number of people expected to flood the biometric system (see Fig. 4b). People not on the watch list are "impostors" like, whose negative identification is sought after. The next experiment reported has 150 subjects, three images from each subject, for a total of 450 face images. We compare the Open Set PCA, Fisherfaces \} and Open Set TCM - kNN classifiers on small watch lists ("galleries"), whose size varies from 10 to 40 subjects, and reports the mean (average) performance (detection and identification) rates obtained over 100 randomized runs. Let the watch list size be $n$ subjects, each of them having 2 (two) images in the gallery. Then there are $450-2 n$ face images in the probe set, $n$ stands for the number of subjects on the watch list and $3 \times(150-n)$ stands for the number of face images that come from subjects that are not on the watch list. The small size of the watch list requires for stability purposes that the rejection threshold be derived from larger populations but still using as before the same statistics of intra- and inter-distance distribution for Open Set $\{$ PCA, Fisherfaces $\}$ and $P S R$ distribution for Open Set TCM-kNN. The decision thresholds $\Theta$ are derived in a manner similar to that used by cohort models in speech (see Sect. 5) by augmenting the gallery with different subjects randomly drawn from other FERET batches that include illumination and facial expression variation. The size of the gallery used to determine the threshold is kept constant at 80 throughout the runs so the number $80-n$ of different subjects needed to augment it varies according to the size $i$ of the watch list.

Table 1 and 2 shows the mean performance of Open Set \{PCA, Fisherfaces\} and Open Set TCM - kNN for different watch list sizes. For watch list size $n$, the accuracy (detection and identification rate) is (average correct rejection + average correct recognition) / $(450-2 n)$. The numerical results, when the number of subjects on the watch list is $n$, should be interpreted as follows. Since the watch list size is much smaller than the number of subjects that should be 
rejected, the (detection and identification rate) accuracy will be very high even if all the probes are rejected. As a consequence the average correct reject number, average correct recognition number, and the detection and identification accuracy are shown for performance evaluation. The average results are better the closer the correct rejection number is to $3 \times(150-n)$, the closer the correct recognition number is to the watch list size, and the higher the accuracy is. Table 1 shows the average performance of Open Set \{PCA, Fisherfaces\} for different watch list sizes. The threshold for rejection is determined from the statistics of intra- and inter-distance distribution (see Sect. 7). The Fisherfaces components outperform PCA components for both rejection and identification decisions. As the watch list size increases, the performance drops.

Table 1. Mean Performance of Open Set $\{$ PCA, Fisherfaces\}

\begin{tabular}{|c|c|c|c|c|c|c|}
\hline \multirow{2}{*}{$\begin{array}{l}\text { Watch } \\
\text { List } \\
\text { Size }\end{array}$} & \multicolumn{3}{|c|}{ Eigenfaces $\left(\mathrm{Mah}+L_{2}\right.$ distance $)$} & \multicolumn{3}{|c|}{ Fisherfaces (Cosine distance) } \\
\hline & $\begin{array}{c}\text { Average } \\
\text { Correct } \\
\text { Reject }\end{array}$ & $\begin{array}{c}\text { Average } \\
\text { Correct } \\
\text { Recognition }\end{array}$ & Accuracy & $\begin{array}{c}\text { Average } \\
\text { Correct } \\
\text { Reject }\end{array}$ & $\begin{array}{c}\text { Average } \\
\text { Correct } \\
\text { Recognition }\end{array}$ & Accuracy \\
\hline 10 & 369.03 & 6.68 & $87.37 \%$ & 389.16 & 7.93 & $92.35 \%$ \\
\hline 15 & 355.67 & 9.82 & $87.02 \%$ & 373.86 & 12.39 & $91.96 \%$ \\
\hline 20 & 343.81 & 12.43 & $86.89 \%$ & 359.92 & 17.04 & $91.94 \%$ \\
\hline 25 & 331.4 & 15.09 & $86.62 \%$ & 345.11 & 20.60 & $91.43 \%$ \\
\hline 30 & 319.5 & 17.79 & $86.48 \%$ & 330.70 & 24.93 & $91.18 \%$ \\
\hline 35 & 305.43 & 20.42 & $85.75 \%$ & 315.15 & 29.04 & $90.58 \%$ \\
\hline 40 & 292.97 & 23.19 & $85.45 \%$ & 300.95 & 32.99 & $90.25 \%$ \\
\hline
\end{tabular}

Table 2 shows the average performance of Open Set TCM-kNN for different watch list sizes. PCA components and the Fisherfaces components yield similar performance when using Open Set TCM - kNN. The Fisherfaces components are a little better than PCA when identification decisions are involved. Open Set TCM-kNN is better than Open Set $\{$ PCA, Fisherfaces\}, when the correct rejection, correct recognition, and the accuracy are taken into account, especially when the watch list size is large. The overall performance for Open Set TCM - kNN, which 
keeps almost constant as the watch list size increases, is thus more stable than the overall performance displayed by Open Set $\{$ PCA, Fisherfaces $\}$.

Table 2. Mean Performance of Open Set TCM-kNN

\begin{tabular}{|c|c|c|c|c|c|c|}
\hline \multirow{2}{*}{$\begin{array}{c}\text { Watch } \\
\text { List } \\
\text { Size }\end{array}$} & \multicolumn{2}{|c|}{ Eigenfaces $\left(\mathrm{Mah}+L_{2}\right.$ distance) } & \multicolumn{2}{c|}{ Fisherfaces (Cosine distance) } \\
\cline { 2 - 7 } & $\begin{array}{c}\text { Average } \\
\text { Correct } \\
\text { Reject }\end{array}$ & $\begin{array}{c}\text { Average } \\
\text { Correct } \\
\text { Recognition }\end{array}$ & Accuracy & $\begin{array}{c}\text { Average } \\
\text { Correct } \\
\text { Reject }\end{array}$ & $\begin{array}{c}\text { Average } \\
\text { Correct } \\
\text { Recognition }\end{array}$ & Accuracy \\
\hline 10 & 389.74 & 7.64 & $92.41 \%$ & 393.08 & 8.48 & $93.62 \%$ \\
\hline 15 & 376.28 & 11.72 & $92.38 \%$ & 380.24 & 12.16 & $93.43 \%$ \\
\hline 20 & 364.18 & 14.12 & $92.27 \%$ & 365.28 & 16.16 & $93.03 \%$ \\
\hline 25 & 350.24 & 18.26 & $92.13 \%$ & 351.84 & 19.56 & $92.85 \%$ \\
\hline 30 & 336.94 & 21.62 & $91.94 \%$ & 335.72 & 25.52 & $92.63 \%$ \\
\hline 35 & 322.96 & 25.22 & $91.63 \%$ & 322.28 & 29.36 & $92.54 \%$ \\
\hline 40 & 309.24 & 27.98 & $91.14 \%$ & 308.64 & 33.28 & $92.41 \%$ \\
\hline
\end{tabular}

The difference in performance between Fig. 7 and Table 2 indicates that the gallery size is also an important factor affecting algorithm performance. In Fig. 7 the gallery (watch list) size is always 75 subjects and only the overlap size between the gallery and probe sets changes, while in Table 2 the gallery size (watch list) varies according to $n$.

\section{Pattern Specific Error Inhomogeneities Analysis (PSEI)}

It is important to know not only what works and to what extent it works, but also to know what does not work and why (Pankanti et al., 2002). Anecdotal evidence suggests that $90 \%$ of errors are due to only $10 \%$ of the face patterns. The contribution made by face patterns to the overall system error is thus not even. Characterization of individual contributions to the overall face recognition system error has received, however, little attention.

Pattern Specific Error Inhomogeneities (PSEI) analysis (Doddington et al, 1998) shows that the error rates vary across the population. It has led to the jocular characterization of the target population as being composed of "sheep" and "goats". In this characterization, the sheep, for 
whom authentication systems perform reasonably well, dominate the population, whereas the goats, though in a minority, tend to determine the performance of the system through their disproportionate contribution of false reject errors. Like targets, impostors also have barnyard appellations, which follow from inhomogeneities in impostor performance across the population. Specifically there are some impostors who have unusually good success at impersonating many different targets. These are called "wolves". There are also some targets that are easy to imitate and thus seem unusually susceptible to many different impostors. These are called "lambs".

ROC can be improved if some of the most difficult data (e.g., the "goats", the hard to match subjects) were excluded and/or processed differently. In general, if the tails of the ROC curve do not asymptote at zero FAR and zero FRR, there is probably some data that could be profitably excluded and maybe processed offline. The trick is to find some automatic way of detecting these poor data items (Bolle et al., 2004) and adopt solutions different from “one size fits all".

We describe next our approach for PSEI analysis that divides the subjects' faces into corresponding "barnyard" classes. The analysis of the error structure in terms of rejection and acceptance decisions follows that of Pankanti et al. (2002) for fingerprints but is driven here by transduction and applied to open set face recognition. There are low matching PSR scores $X$ that, in general, do not generate false rejects, and there are high matching PSR scores $Y$ associated with subjects that generally do not generate false accepts. The corresponding "rejection / mismatch" and "acceptance / match" cumulative distributions for some rejection threshold $\boldsymbol{\Theta}=T$ are $F^{T}$ and $G^{T}$ :

$$
\begin{aligned}
& F^{T}(x)=\#(P S R \leq x \mid P S R \leq T) / \#(P S R \leq T) \\
& G^{T}(\mathrm{y})=\#(P S R \geq y \mid P S R>T) / \#(P S R>T)
\end{aligned}
$$


As the scores $X$ and $Y$ are samples of ordinal random variables, the Kolmogorov - Smirnov (KS) measure (Conover, 1980) compares the individual score distributions $F_{i}(x)$ and $G_{i}(\mathrm{y})$ (see below) for subject $i$ with the (typical) distributions $F^{T}$ and $G^{T}$, respectively.

$$
\begin{gathered}
F_{i}(x)=\#(P S R \leq x \mid P S R \text { from rejected subject } i) / \#(P S R \text { from rejected subject } i) \\
G_{i}(\mathrm{y})=\#(P S R \geq y \mid P S R \text { from accepted subject } i) / \#(P S R \text { from accepted subject } i)
\end{gathered}
$$

The distances between the individual and typical distributions for the KS test are $\left|\Delta_{i}^{F}\right|$ and $\left|\Delta_{i}^{G}\right|$, respectively, and they quantify the variance in behavior for subject $i$ from the typical behavior. The unsigned $\Delta_{i}^{F}$ and $\Delta_{i}^{G}$ quantities, however, are used for PSEI analysis because they express how well subject i's match PSR scores agree with well-behaved, easy to correctlyreject subjects, and with well-behaved, easy to correctly recognize subjects, respectively.

$$
\begin{aligned}
& \Delta_{i}^{F}=F_{i}\left(x_{\max }\right)-F^{T}\left(x_{\max }\right) \text { where } x_{\max }=\arg \max \left|F_{i}(x)-F^{T}(x)\right| \\
& \Delta_{i}^{G}=G_{i}\left(y_{\max }\right)-G^{T}\left(y_{\max }\right) \text { where } y_{\text {max }}=\arg \max \left|G_{i}(y)-G^{T}(y)\right|
\end{aligned}
$$

Negative (positive) $\Delta$ indicates that the average population score belonging to some subject $i$ is lower (higher) than the average (well-behaved) overall population. The identities left (right) of the $y$ - axis display undesirable (desirable) intra-pattern similarity. Similarly, identities above (below) $x$ - axis display desirable (undesirable) inter - pattern similarity. Many identities clustering along the axes imply that co - existence of both (un)desirable match score property and (un) desirable non-match property is very unlikely. Most of the population shows either a central (near origin) tendency or a tendency to deviate along one of the axis. For rejection, positive (negative) $\Delta_{i}^{F}$ implies that the average rejection PSR for subject $i$ is higher (lower) than the average rejection $P S R$ for the whole population. For acceptance, negative (positive) $\Delta_{i}^{G}$ implies that the average acceptance $P S R$ for subject $i$ is higher (lower) than the average acceptance $P S R$ for the whole population. 


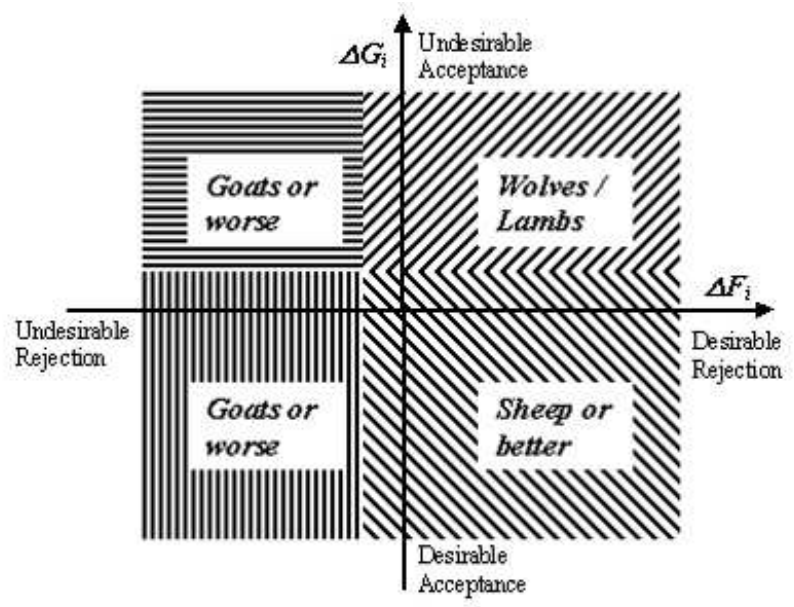

(a) Zoo Display

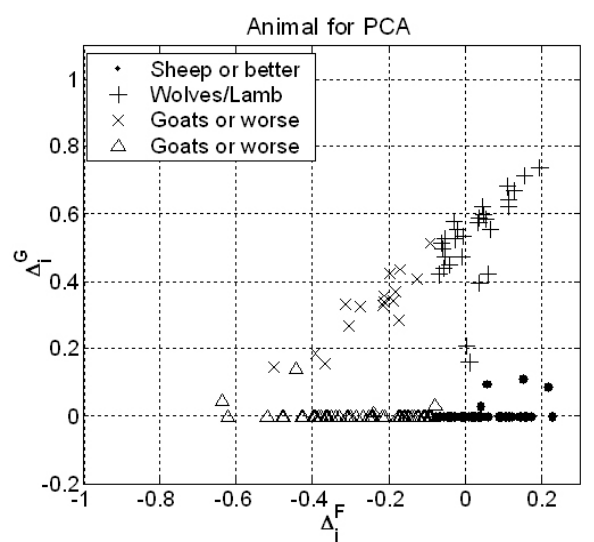

(b) Open Set TCM-kNN "PCA"

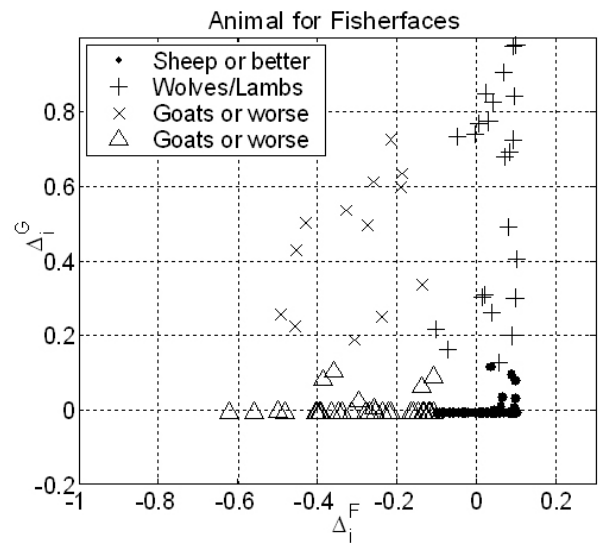

(c) Open Set TCM-kNN "Fisherfaces"

Figure 9. Variant Behavior for Rejection and Acceptance

The different types of animals are located in Fig. 9a using the four-quadrant diagram induced by the 2D scatter plot of $\left(\Delta_{i}^{F}, \Delta_{i}^{G}\right)$. In general, the more negative $\Delta_{i}^{F}$ is, the more false acceptance subject $i$ accounts for; the more positive $\Delta_{i}^{G}$ is, the more false rejection subject $i$ accounts for. One threshold has to be chosen for deciding what counts as desirable or undesirable behavior. If one assumes that the desirable behavior subject set accounts only for $1 \%$ false rejection or false acceptance, one can sort $\Delta_{i}^{F}$ and $\Delta_{i}^{G}$ and determine the thresholds $T_{\mathrm{F}}$ and $T_{\mathrm{G}}$ such that (i) all subjects for whom $\Delta_{i}^{F} \geq T_{\mathrm{F}}$ account only for $1 \%$ false acceptance and (ii) all subjects for whom $\Delta_{i}^{G} \leq T_{\mathrm{G}}$ account only for $1 \%$ false acceptance. Most of the sheep occupy the forth 
quadrant and are characterized by desirable rejection behavior when $\Delta_{i}^{F}$ is greater than $T_{\mathrm{F}}$ and desirable acceptance behavior when $\Delta_{i}^{G}$ is less than $T_{\mathrm{G}}$. Note that if the PSR value of the probe is far away from the threshold, the decisions are easy to make; only the parts of the distributions near thresholds are likely to contribute to errors and need to be considered. The first quadrant is characteristic of subjects that display desirable rejection behavior (when they should be indeed rejected) but also of subjects showing undesirable rejection behavior (when they should be accepted) (wolves / lambs). The second quadrant includes subjects that are difficult to label in terms of rejection or recognition (goats). The third quadrant is characteristic of subjects that display desirable acceptance behavior (when they should be accepted) but also of subjects showing undesirable acceptance behavior when they should be instead rejected (goats). Finally, the fourth quadrant represents subjects with good performance (sheep).

Open Set TCM - kNN using either PCA or Fisherfaces components is run 1,000 times to yield the corresponding PSR scores. The overlap, of size $\mathrm{m}=40$, between the gallery and test sets of size 80 , is randomly chosen. The 2D scatter plots (for 150 subjects) of $\left(\Delta_{i}^{F}, \Delta_{i}^{G}\right)$ for Open Set TCM-kNN using PCA and Fisherfaces, respectively, are shown in Figs. 9b and 9c, respectively. Most of the subjects show a central (near origin) tendency with good performance. Several subjects show the tendency to deviate along one of the axis. Only few subjects show undesirable characteristics along both axes. The average false rejection and false acceptance rates for Open Set TCM - kNN using PCA components are $13.36 \%$ and $8.29 \%$, respectively. The subjects with the top $10(20) \Delta_{i}^{G}$ values contribute $24.76 \%$ (50.09\%) of total false rejection. The subjects with top $10(20)-\Delta_{i}^{F}$ values contribute $28.54 \%(49.85 \%)$ of total false acceptance. The average false rejection and false acceptance rates for Open Set TCM - kNN using Fisherfaces are $8.33 \%$ and $4.37 \%$, respectively. The subjects with top $10(20) \Delta_{i}^{G}$ values contribute $32.19 \%(62.64 \%)$ of 
total false rejection. The subjects with top $10(20)-\Delta_{i}^{F}$ values contribute $38.74 \%(68.83 \%)$ of total false acceptance.

The decision threshold is determined based on the above discussion for both PCA and Fisherfaces components. For PCA, there are 52 (34.67\%) subjects classified as Sheep or better, $28(18.67 \%)$ as Wolves/Lambs, and $54(36 \%)$ as Goats or worse in the third quadrant. Only 16 (10.67\%) subjects are classified as Goats or worse in the second quadrant, and they contribute to both $22.92 \%$ false rejection and $20.94 \%$ false acceptance. $37 \%$ of subjects are error prone animals (not sheep) and they contribute about $98.2 \%$ and $98.9 \%$ of the total false rejection and false acceptance errors, respectively. For Fisherfaces, there are $78(52 \%)$ subjects classified as Sheep or better, 24 (16\%) as Wolves/Lambs, and 35 (23.3\%) as Goats or worse in the third quadrant. Only $13(8.67 \%)$ subjects are classified as Goats or worse in the second quadrant, and they contribute to both $32.1 \%$ false rejection and $29.0 \%$ false acceptance. $48 \%$ of subjects are error prone animals (not sheep), and they contribute about $98.9 \%$ and $98.5 \%$ of total false rejection and false acceptance errors, respectively. All the error prone animals contribute to either false rejection or false acceptance for both PCA and Fisherfaces components. If some error prone animals are removed from the test set, the performance for Open Set TCM-kNN will improve. As an example, if all Goats or worse in the second quadrant are removed for PCA and Fisherfaces, and the experiments in Sect. 7 are redone, the Open Set TCM-kNN performance improves and it now achieves $85.69 \%$ and $91.63 \%$ accuracy (at FAR $=3 \%$ ) for PCA and Fisherfaces, respectively, vs. the earlier results of $81 \%$ and $88 \%$ accuracy (at FAR $=6 \%$ ).

\section{Data Fusion}

The overlap in labeling between Open Set TCM-kNN \{PCA and Fisherfaces\} regarding error prone animals discussed in the previous section reveals useful insights about their comparative 
contributions and suggests the possibility to fuse their outputs for enhanced authentication. Note that data fusion for the outputs of different face recognition engines is a particular case of information fusion (Ross and Jain, 2004) for multi(modal)-biometric systems (Jain and Ross, 2004) where different modalities, e.g., fingerprints, face and hand geometry, are used. Multimodal biometrics that combine face and fingerprints have been shown to yield significant performance improvement over single-mode biometric systems (Snelick et al., 2003). We describe next how multi-system fusion using Open Set TCM-kNN \{PCA and Fisherfaces $\}$ driven by PSEI analysis yields better performance for authenticating those subjects that are rejected by one classifier and accepted by another classifier.

Assume that during Open Set TCM-kNN the thresholds $\boldsymbol{\Theta}$, the training PSR standard deviations, the probe PSR values and confidences for PCA and Fisherfaces are $\boldsymbol{\Theta}_{\text {PCA }}$ and $\boldsymbol{\Theta}$ Fisher, $S_{\text {Stdev }}{ }_{\mathrm{PCA}}$ and $S_{\text {tdev }}$ Fisher, $_{\text {PSA }}$ and PSR ${ }_{\text {Fisher, }} C_{\mathrm{PCA}}$ and $C_{\text {Fisher, }}$ respectively. The first case occurs when the probe is accepted if both $P S R_{\mathrm{PCA}} \geq \boldsymbol{\Theta}_{\mathrm{PCA}}$ and $P S R_{\text {Fisher }} \geq \boldsymbol{\Theta}_{\text {Fisher. The }}$ identification of the probe is then determined as follows: (i) If Open Set TCM-kNN \{PCA and Fisherfaces $\}$ yield the same ID for their largest (credibility) $p$-values, then the decision taken is to authenticate the ID no matter what the confidences are; and (ii) If the Open Set TCM-kNN PCA, Fisherfaces $\}$ yield different ID for their largest $p$-values, then choose that ID that yields larger confidence. In the case when the two confidences are very close to each other, choose the label coming from Open Set TCM-kNN Fisherfaces classifier because it yields better performance than Open Set TCM-kNN PCA classifier (see Sect. 7).

The second case occurs when the probe is rejected if both $P S R_{\mathrm{PCA}}<\boldsymbol{\Theta}_{\mathrm{PCA}}$ and $P S R_{\text {Fisher }}<\boldsymbol{\Theta}$ Fisher. The third possible case occurs when $P S R_{\mathrm{PCA}} \geq \boldsymbol{\Theta}_{\mathrm{PCA}}$ and $P S R_{\text {Fisher }}<\boldsymbol{\Theta}_{\text {Fisher, }}$ i.e., the two engines disagree if rejection should take place. One has now to consider how far the probe PSRS 
are away from the thresholds $\boldsymbol{\Theta}$ and the relative location of the class predicted by Open Set TCMKNN PCA in the zoo. When the label predicted by Open Set TCM-KNN PCA is a sheep, the probe is accepted and its ID is that predicted. If the label predicted by Open Set TCM-kNN PCA is not a sheep then compare the following distances $\alpha=\left(P S R_{\mathrm{PCA}}-\Theta_{\mathrm{PCA}}\right) / \operatorname{Stdev}_{\mathrm{PCA}}$ and $\beta=(\boldsymbol{\Theta}$

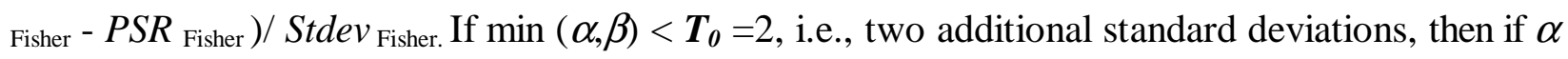
$>\beta$ the ID that Open Set TCM-kNN PCA predicts is accepted; otherwise the probe is rejected. If $\min (\alpha, \beta)>T_{0}$, the probe is rejected using the very decision Open Set TCM-kNN Fisherfaces classifier makes. Similar arguments apply for the last case when $P S R_{\mathrm{PCA}}<\boldsymbol{\Theta}_{\mathrm{PCA}}$ and $P S R_{\text {Fisher }} \geq$ $\boldsymbol{\Theta}_{\text {Fisher. }}$ Using the data fusion procedure described above, the experiments in Sect. 7 were redone to yield now $91.55 \%$ accuracy and only $3.1 \%$ false alarm vs. $81 \%$ and $88 \%$ correct accuracy and false alarm of $6 \%$ without fusion. Note that one achieves $91 \%$ accuracy without excluding any error prone exemplar (see Sect. 9). Note that PCA and Fisherfaces are not independent representations. Some animals were observed to continue to be error prone after the LDA step (of Fisherfaces) once they were bad for PCA. In our data set, only 33 subjects are sheep for both PCA and Fisherfaces components, while 64 subjects are sheep for either PCA or Fisherfaces components.

\section{Conclusions}

This paper expands on the Transduction Confidence Machine (Proedrou, 2001) to make it suitable for the open set recognition problem. Towards that end we introduced the Open Set TCM - kNN (Transduction Confidence Machine - k Nearest Neighbors), a novel realization of transductive inference that is suitable for open set multi-class classification and includes a rejection option. Extensive experimental data, using challenging FERET data, shows the comparative advantages of Open Set TCM-kNN. The major contributions made include multi- 
class transductive inference using a priori threshold setting, effective open set identification and watch list, meaningful error analysis to determine the uneven contributions to errors made by different face patterns and their use for effective data fusion.

The proposed rejection functionality for open set recognition is similar to that used in detection theory, hypothesis testing, and score normalization (See Sect. 5). The availability of the rejection option, i.e., "none of the above" answer, in open set recognition, is similar to outlier detection (in clustering) and novelty detection, topics of further investigation. The comparative advantages of our proposed method come from its non-parametric implementation and automatic threshold selection. No assumptions are made regarding the underlying probability density functions responsible for the observed data clusters, i.e., the face IDs. Learning and training, driven by transduction, are local. They provide robust information to detect outlier faces, i.e., unknown faces, and to reject them accordingly. Outlier detection corresponds to change detection when faces or patterns change their appearance. Feature selection for enhanced pattern recognition can be further achieved using strangeness and the p-value function. The stranger the feature values are the better the discrimination between the patterns.

The acquisition and/or generation of additional exemplars for each class (to increase $k$ in Open Set TCM-kNN) is presently under investigation and is expected to lead to improved performance. Another direction for future research concerns taking advantage of the linkage between transductive inference (see Sects. 3 - 5), active learning, co-training, and normalization methods. The active learner has to decide whether or not to request labels ("classification") for unlabeled data in order to reduce the volume of computation and the human effort involved in labeling (Tong and Keller, 2001). Active learning selects, one by one, the most informative patterns from some working set $W$, such that, after labeling by an expert ("classifier"), they will guarantee the best improvement in the classifier performance. As an example, the sampling strategy proposed by 
Juszczak and Duin (2004) relies on measuring the variation in label assignments (of the unlabeled set) between the classifier trained on $T$ and the classifier trained on $T$ with a single unlabeled exemplar $\boldsymbol{e}$ labeled with all possible labels. The use of unlabeled data, independent of the learning algorithm, is characteristic of co-training (Blum and Mitchell, 1998; Nigam et al., 2000). The idea of co-training is to learn two classifiers which bootstrap each other using labels for the unlabeled data (Krogel and Scheffer, 2004). Co-training leads to improved performance if at least one classifier labels at least one unlabeled instance correctly for which the other classifier currently errs. Unlabeled examples which are confidently labeled by one classifier are then added, with labels, to the training set of the other classifier.

\section{References}

[1].E. Bailly-Bailliere et al. (2003), The BANCA Database and Evaluation Protocol, 4th AudioVideo Based Person Authentication (AVBPA), 625 - 638.

[2].S. Bengio, J. Mariethoz, and S. Marcel (2001), Evaluation of Biometric Technology on XM2VTS, IDIAP-RR 01-21, European Project BANCA Deliverable D71, Martigny, Switzerland.

[3].A. Blum and T. Mitchell (1998), Combining Labeled and Unlabeled Data with Co-Training, in COLT: Proc. of the Conf. on Computational Learning Theory, 92 - 100, Morgan Kaufmann.

[4].R. M. Bolle, J. H. Connell, S. Pankanti, N. K. Ratha, and A. W. Senior (2004), Guide to Biometrics, Springer.

[5].R. Chellappa, C. L. Wilson and S. Sirohey (1995), Human and Machine Recognition of Faces: A Survey, Proc. IEEE, Vol. 83, No. 5, $705-740$.

[6].T. M. Cover and P. E. Hart (1967), Nearest neighbor pattern classification, IEEE Trans. Inform. Theory IT 13:21-7.

[7].P. Grother (2004), Face Recognition Vendor Test 2002, Supplemental Report-NISTIR 7083. 
[8].J. Daugman (1997), Face and Gesture Recognition: Overview, IEEE Trans. on PAMI, Vol. 19, 7, $675-676$.

[9]. G. R. Doddington, W. Liggett, A. Martin, M. Przybocki and D. Reynolds (1998), Sheep, Goats, Lambs and Wolves: A Statistical Analysis of Speaker Performance, Proc. Of ICSLD'98, 1351-1354.

[10]. S. Furui (1997), Recent Advances in Speaker Recognition, Pattern Recognition Letters, 18, $859-872$.

[11]. A. Gammerman, V. Vovk, and V. Vapnik (1998), Learning by Transduction. In Uncertainty in Artificial Intelligence, 148 - 155.

[12]. A. Jain, R. Bolle, and S. Pankanti (Eds.) (1999), BIOMETRICS - Personal Identification in Networked Society, Kluwer.

[13]. A. Jain and A. Ross (2004), Multibiometric Systems, Comm. of ACM, Vol.47, No.1,34- 40.

[14]. T. Joachims (1999), Transductive Inference for Text Classification Using Support Vector Machines, in I. Bratko and S. Dzeroski (eds.), Proc. Of ICML-99, 16th Int. Conf. on Machine Learning, Bled, Slovenia, Morgan Kaufmann, 200 - 209.

[15]. P. Juszczak and R. P. W. Duin (2004), Selective Sampling Based on the Variation in Label Assignment, 17th Int. Conf. on Pattern Recognition (ICPR), Cambridge, England.

[16]. M. A. Krogel and T. Scheffer (2004), Multi-Relational Learning, Text Mining, and SemiSupervised Learning from Functional Genomics, Machine Learning, 57, 61 - 81.

[17]. M. Kukar and I. Kononenko (2002), Reliable Classifications with Machine Learning, 13th European Conf. on Machine Learning (ECML), Helsinki, Finland.

[18]. M. Li and P. Vitanyi (1997), An Introduction to Kolmogorov Complexity and Its Applications, 2nd. Springer-Verlag. 
[19]. C. Liu and H. Wechsler (2002), Gabor Feature Based Classification Using the Enhanced Fisher Linear Discriminant Model for Face Recognition, IEEE Trans. on Image Processing, Vol. 11, No. 4, 467 - 476.

[20]. C. Liu and H. Wechsler (2004), Facial Recognition in Biometric Authentication: Technologies, Systems, Evaluations and Legal Issues, J.L.Wayman, A. Jain, D. Maltoni and D. Maio (Eds.), Springer-Verlag (to appear).

[21]. M.W. Mak, W.D. Zhang and M.X. He (2001), A New Two-Stage Scoring Normalization Approach to Speaker Verification," Proc. Int. Sym. on Intelligent Multimedia, Video and Speech Processing, pp. 107-110, Hong Kong.

[22]. T. Melluish, C. Suanders, I. Nouretdinov, I and V. Vovk (2001), The Typicalness Framework: A Comparison with the Bayesian Approach. TR, Dept. of Computer Science, Royal Holloway, University of London, http://www.clrc.rhul.ac.uk/tech-report/.

[23]. T. Mitchell (1999), The Role of Unlabelled Data in Supervised Learning, Proc. 6th Int. Colloquium on Cognitive Sciences, San Sebastian, Spain.

[24]. K. Nigam, A. K. McCallum, S. Thrun and T. M. Mitchell (2000), Text Classification from Labeled and Unlabeled Documents Using EM, Machine Learning, 39 (2/3), 103 - 134.

[25]. S. Pankanti, N. K. Ratha and R. M. Bolle (2002), Structure in Errors: A Case Study in Fingerprint Verification, 16th Int. Conf. on Pattern Recognition, Quebec-City, Canada.

[26]. P. J. Phillips, H. Wechsler, J. Huang, and P. Rauss (1998), The FERET Database and Evaluation Procedure for Face Recognition Algorithms, Image and Vision Computing, Vol. 16, No. 5, 295- 306 .

[27]. P. J. Phillips, P. Grother, R. J. Micheals, D. M. Blackburn, E. Tabassi and M. Bone (2003), Face Recognition Vendor Test 2002 - Overview and Summary. 
[28]. K. Proedrou, I. Nouretdinov, V. Vovk and A. Gammerman (2001), Transductive Confidence Machines for Pattern Recognition, TR CLRC-TR-01-02, Royal Holloway University of London.

[29]. D. A. Reynolds, T. F. Quatieri, and R. B. Dunn (2000), Speaker Verification Using Adapted Gaussian Mixture Models, Digital Signal Processing 10, 19 - 41.

[30]. A. Ross and A. Jain (2004), Information fusion in Biometrics, Pattern Recognition Letters, Vol. 24, $2115-2125$.

[31]. C. Saunders, A. Gammerman, and V. Vovk (1999), Transduction with Confidence and Credibility, 16th Int. Joint Conf. on Artificial Intelligence (IJCAI), Stockholm, Sweden.

[32]. R. Snelick, M. Indovina, J. Yen, and A. Mink (2003), Multimodal Biometrics: Issues in Design and Testing, ICMI'03, Vancouver, BC, Canada.

[33]. S. Tong and D. Koller (2001), Support Vector Machines Active Learning with Applications to Text Classification, Journal of Machine Learning Research, Vol. 2, 45 - 66

[34]. V. N. Vapnik (1998), Statistical Learning Theory, Wiley.

[35]. V.N. Vapnik (2000), The Nature of Statistical Learning Theory, 2nd. Ed., Springer-Verlag.

[36]. W.J.Conover, Practical Nonparametric Statistics, John Wiley \& Sons, Inc. 1980.

[37]. V. Vovk, A. Gammerman and C. Saunders (1999), Machine-Learning Application of Algorithmic Randomness, Proceedings of the1 6th International Conference on Machine Learning, Bled, Slovenia.

[38]. W. Zhao, R. Chellappa, P. J. Phillips, and A. Rosenfeld (2003), Face Recognition: A Literature Survey, Computing Surveys, Vol. 35, No. 4, 399 - 458. 